\title{
Flora da Reserva Biológica Guaribas, Estado da Paraíba, Brasil: Erythroxylaceae ${ }^{1}$
}

\author{
James Lucas da Costa-Lima ${ }^{2,3}$ e Marccus Alves ${ }^{3,4}$
}

Recebido: 14.11.2017; aceito: 18.05.2018

\begin{abstract}
Flora of the Reserva Biológica Guaribas, Paraíba State, Brazil: Erythroxylaceae). Erythroxylaceae comprises between 240-250 species in the tropical areas of the globe, almost all of them belonging to the genus Erythroxylum. The greatest diversity of the genus is in the Neotropics, with Brazil as the main center of diversity and endemism. In Brazil there are 128 species, of which 83 are endemic, with greater richness in the Atlantic Forest Domain, both in the coastal forests and in their associated ecosystems, especially in northeastern Brazil. The Erythroxylaceae florula presented here is part of the floristic survey of the Reserva Biológica Guaribas, in the State of Paraíba, an important protected area of tabuleiro savanna vegetation in northeastern Brazil. This study was based on the analysis of samples deposited at CEN, EAN, HUEFS, JPB, RB, UFP, and UFRN herbaria, as well as fieldwork in the study area. Three species of Erythroxylum were confirmed to the area: Erythroxylum passerinum Mart., E. rimosum O.E.Schulz and E. simonis Plowman. An identification key, morphological descriptions, comments on geographical distribution, preferential habitats and illustrations of the main diagnostic features of the species are provided.
\end{abstract}

Keywords: Atlantic Forest, Erythroxylum, floristics, Malpighiales, taxonomy

RESUMO - (Flora da Reserva Biológica Guaribas, Estado da Paraíba, Brasil: Erythroxylaceae). Erythroxylaceae compreende entre 240-250 espécies distribuídas nas áreas tropicais do globo, quase todas pertencentes ao gênero Erythroxylum. A maior diversidade do gênero está na região Neotropical, tendo o Brasil como principal centro de diversidade e endemismo. No Brasil são registradas 128 espécies, das quais 83 são endêmicas, com maior riqueza no Domínio da Mata Atlântica, tanto na floresta costeira quanto nos ecossistemas associados, especialmente na Região Nordeste do Brasil. A flórula de Erythroxylaceae aqui apresentada é parte do levantamento florístico da Reserva Biológica (REBIO) Guaribas, no Estado da Paraíba, uma importante Unidade de Conservação nos tabuleiros litorâneos do Nordeste do Brasil. O estudo foi baseado na análise de materiais depositados nos herbários CEN, EAN, HUEFS, JPB, RB, UFP e UFRN, além de coletas realizadas na área de estudo. Na REBIO Guaribas foi confirmada a ocorrência de três espécies de Erythroxylum, a saber: Erythroxylum passerinum Mart., E. rimosum O.E.Schulz e E. simonis Plowman. O trabalho consta de chave analítica, descrições morfológicas, comentários sobre distribuição geográfica e hábitats preferenciais e ilustrações dos principais caracteres diagnósticos das espécies.

Palavras-chave: Erythroxylum, florística, Malpighiales, Mata Atlântica, taxonomia

\section{Introdução}

Erythroxylaceae (Malpighiales) possui distribuição pantropical e compreende quatro gêneros e cerca de 250 espécies, dez delas correspondem aos gêneros africanos Aneulophus Benth., Nectaropetalum Engl. e Pinacopodium Exell \& Mendonça e as demais espécies a Erythroxylum P.Browne (Daly 2004).
Embora tenha distribuição pantropical, a maior parte das espécies de Erythroxylum (ca. 200 spp.) é endêmica a região Neotropical (Plowman \& Hensold 2004).

Erythroxylum é amplamente conhecido pela produção de alcaloides de grande importância econômica, como a cocaína e a catuabina (Daly 2004). Suas espécies são caracterizadas por

1. Parte da Dissertação de Mestrado do primeiro Autor

2. Universidade Estadual de Feira de Santana, Departamento de Ciências Biológicas, Herbário HUEFS, Avenida Transnordestina, s/n, Novo Horizonte, 44036-900 Feira de Santana, BA, Brasil

3. Universidade Federal de Pernambuco, Centro de Biociências, Departamento de Botânica, Avenida Professor Moraes Rego, s/n, 50670-420 Recife, PE, Brasil

4. Autor para correspondência: jimcostalima@yahoo.com.br 
serem árvores ou arbustos glabros, com ramos geralmente lenticelados, estípulas intrapeciolares, catafilos em geral concentrados formando ramos encurtados (braquiblastos), folhas simples, alternas, inflorescências em cimas axilares, em geral congestas, flores pentâmeras, heterostílicas e frutos do tipo drupa com um único pirênio com morfologia variada (Schulz 1907, Plowman \& Berry 1999, Costa-Lima \& Alves 2015a). No Brasil são registradas 128 espécies de Erythroxylum, colocando o país como principal centro de riqueza e endemismo do gênero (Daly 2004, Loiola \& Costa-Lima 2015). Destas, 83 espécies são restritas ao Domínio da Mata Atlântica e a maioria delas ocorre na Região Nordeste do Brasil (Plowman 1987, Loiola \& Costa-Lima 2015), que é a porção mais fragmentada desse Domínio (Ranta et al. 1998). Loiola et al. (2007) registraram 13 espécies no Estado da Paraíba e, posteriormente, Loiola \& Costa-Lima (2015) apresentaram 15 espécies numa listagem mais atualizada, sendo a maioria delas com distribuição em áreas de Mata Atlântica, especialmente nas restingas, tabuleiros litorâneos e florestas estacionais.

A Reserva Biológica (REBIO) Guaribas está entre as principais unidades de conservação de proteção integral do Estado da Paraíba, cujo remanescente de Mata Atlântica contempla, principalmente, os chamados tabuleiros litorâneos (IBAMA 2003). Apesar da sua importância no contexto da Mata Atlântica do Nordeste do Brasil, a REBIO Guaribas é pouco conhecida no que diz respeito à sua flora $\mathrm{e}$, mesmo tendo uma listagem de plantas para a área (Barbosa et al. 2011), apenas as famílias Rubiaceae (Pereira \& Barbosa 2004, 2006) e Boraginaceae (Melo \& Vieira 2017) foram estudadas mais acuradamente. Sendo assim, aqui é apresentado o tratamento taxonômico das Erythroxylaceae para a REBIO Guaribas como contribuição ao conhecimento da flora dessa Unidade de Conservação.

\section{Material e métodos}

Área de estudo - A Reserva Biológica (REBIO) Guaribas está situada nos municípios de Mamanguape, com mais de $90 \%$ de sua extensão, e Rio Tinto, no Estado da Paraíba, e tem sua área dividida em três unidades: SEMA 1, SEMA 2 e SEMA 3, totalizando $4.028,55$ ha (IBAMA 2003) (figura 1). Seguindo a compilação de Thomas \& Barbosa (2008), a vegetação da REBIO Guaribas apresenta as seguintes fitofisionomias: (i) os tabuleiros litorâneos, que são áreas savânicas em solos da Formação Barreiras, presentes principalmente nas SEMA 1 e 2; e (ii) as florestas estacionais semidecíduas de terras baixas, que compreendem as florestas costeiras em baixas elevações, presentes principalmente na SEMA 2 e 3.

Estudo taxonômico - O estudo foi baseado, principalmente, na análise dos materiais depositados no Herbário JPB e adicional consulta às amostras depositadas nas coleções dos herbários CEN, EAN, HUEFS, RB, UFP e UFRN. As atividades de campo ocorreram no ano de 2013 por meio de coletas aleatórias nas principais áreas da REBIO Guaribas. O processamento das amostras seguiu metodologia usual em taxonomia vegetal (e.g., Mori et al. 1989), com posterior inclusão no acervo do Herbário UFP e duplicatas distribuídas ao HUEFS, JPB, NY, RB e UFRN. Todos os acrônimos de coleções citadas de acordo com Thiers (2018, continuamente atualizado). A identificação das amostras foi realizada por meio de análise comparativa com descrições originais (i.e., Martius 1840, Schulz 1907, Plowman 1986) e os termos morfológicos utilizados nas descrições foram baseados em Stearn (1992) e Harris \& Harris (2001), tendo como base as descrições encontradas em Schulz (1907) e Plowman (1987). A terminologia adotada para os coléteres e pirênios segue a mesma empregada por Costa-Lima et al. (2014). As ilustrações foram confeccionadas a partir do material herborizado coletado pelo primeiro autor. As fenofases reprodutivas foram obtidas com base nos materiais examinados, observações em campo e amostras de outras áreas do Estado da Paraíba. A distribuição

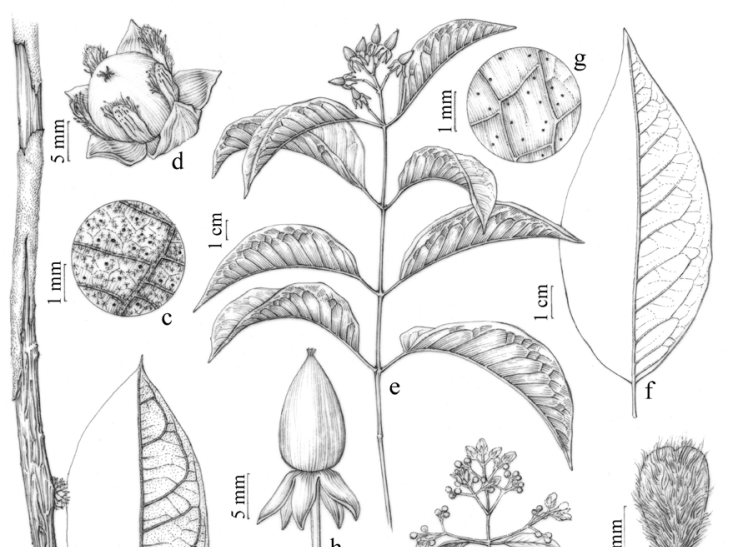

Figura 1. Mapa de localização da Reserva Biológica (REBIO) Guaribas, Estado da Paraíba, Nordeste do Brasil, e suas áreas. Mapa elaborado por Rafael Barbosa da Silva.

Figure 1. Location map of the Guaribas Biological Reserve (REBIO), State of Paraíba, northeastern Brazil, and its areas. Map by Rafael Barbosa da Silva. 
global das espécies foi obtida a partir de bibliografia especializada (e.g., Loiola \& Costa-Lima 2015) e o estado de conservação das espécies foi avaliado seguindo os critérios e categorias da IUCN (2012), utilizando-se da ferramenta GeoCAT (Bachman et al. 2011)<http://geocat.kew.org>, com Área de Ocupação baseada em grades de $2 \mathrm{Km}^{2}$.

\section{Resultados e Discussão}

$\mathrm{Na}$ listagem inicial elaborada por Barbosa et al. (2011), seis espécies de Erythroxylum foram reportadas para a área: Erythroxylum citrifolium A.St.Hil., E. passerinum Mart., E. pauferrense Plowman, E. rimosum O.E.Schulz, E. suberosum var. denudatum O.E.Schulz e E. squamatum Sw. Estas identificações foram checadas e várias delas se revelaram incorretas. Os materiais originalmente identificados como $E$. suberosum var. denudatum correspondem a $E$. rimosum e todos os demais a E. passerinum. Além dessas duas espécies, amostras de E. simonis foram coletadas posteriormente. Desse modo, o gênero Erythroxylum está representado na REBIO Guaribas por três espécies: Erythroxylum passerinum, E. rimosum e E. simonis. Erythroxylum rimosum, que também ocorre em vegetação de carrasco (Domínio da Caatinga), foi encontrada nos tabuleiros litorâneos (Domínio da Mata Atlântica), já E. passerinum e $E$. simonis são espécies restritas à Mata Atlântica (Loiola \& Costa-Lima 2015). Por apresentarem distribuição ampla, nenhuma das três espécies apresenta grau de ameaça segundo os critérios da IUCN (2012), com todas se enquadrando na categoria Pouco Preocupante (LC).

\section{Erythroxylum P.Browne}

Árvores ou arbustos glabros, geralmente com ramos lenticelados, coléteres presentes nas gemas, estípulas, bractéolas e/ou lobos do cálice; folhas alternas, estípulas intrapeciolares, estriadas longitudinalmente ou não, 2-3-setulosas no ápice ou sétulas ausentes; catafilos persistentes, por vezes congestos formando ramos encurtados (braquiblastos); inflorescências cimosas, fasciculadas ou evidentemente pedunculadas, unifloras ou com flores congestas; bractéolas 1 a numerosas; flores pentâmeras, andróginas, heterostílicas; cálice com prefloração contorcida ou valvar; pétalas com apêndice ligulado internamente, com 1-2 pares de aurículas; estames 10, em uma ou duas séries de acordo com o morfo floral, filetes unidos na base; ovário súpero, 3-carpelar, 3-locular, com apenas 1 óvulo desenvolvido; estiletes 3 , livres ou parcialmente concrescidos; drupas carnosas, epicarpo vermelho, purpúreo ou enegrecido; pirênio 1, cilíndrico, trígono ou sulcado longitudinalmente em secção transversal.

Chave para identificação das espécies de Erythroxylaceae da REBIO Guaribas, Paraíba, Brasil

1. Folhas oblongas a obovadas, coriáceas, ápice obtuso, margem finamente revoluta; cálice com prefloração contorcida, lobos oblongos, ápice apiculado .... 2. E. rimosum

1. Folhas estreito a largamente elípticas, membranáceas a cartáceas, ápice acuminado a agudo, margem plana; cálice com prefloração valvar, lobos triangulares, ápice agudo

2. Folhas cartáceas, opacas na superfície adaxial; estípulas comcoléteresinconspícuos a curtamente filamentosos; tubo estaminal menor que os lobos do cálice; drupa com pirênio sulcado longitudinalmente em secção transversal ................................. 1. E. passerinum

2. Folhas membranáceas, lúcidas na superfície adaxial; estípulas com coléteres levemente vilosos; tubo estaminal maior que os lobos do cálice; drupa com pirênio cilíndrico em secção transversal ..... 3. E. simonis

1. Erythroxylum passerinum Mart., Abh. Math.-Phys. Cl. Königl. Bayer. Akad. Wiss. 3(2): 386. 1840. Figura 2 a-d

Arbustos a árvores, 2-8 $\mathrm{m}$ alt.; ramos densamente lenticelados, castanho-acinzentados. Estípulas 2,2-3 mm compr., coléteres inconspícuos a conspícuos e curtamente filamentosos. Pecíolo 1-2 mm compr.; folhas com lâmina 3-5,5 × 1,5-3,6 cm, elípticas a largamente elípticas, cartáceas, opacas em ambas as superfícies, base aguda, margem plana, ápice agudo. Fascículos com 1-6 flores por nó; pedicelo 3,3-6,2 mm compr.; cálice com prefloração valvar, lobos 1-1,4 × 0,8-1,3 mm, triangulares, ápice agudo; pétalas 2,8-4,4 mm compr.; tubo estaminal 0,8-1,2 mm compr.; filetes 1-1,8 mm compr.; estiletes 1,8-2,6 mm compr., livres; ovário 1-1,4 × 0,6-0,8 mm, obovoide. Drupas com pirênio 7-9,5 × 4-6 mm, elipsoide, sulcado longitudinalmente em secção transversal.

Material examinado: BRASIL. Paraíba: Mun. Mamanguape, Reserva Biológica Guaribas, 27-IV-1993 (fl.), M.R. Barbosa 1349 (JPB); ibidem, 

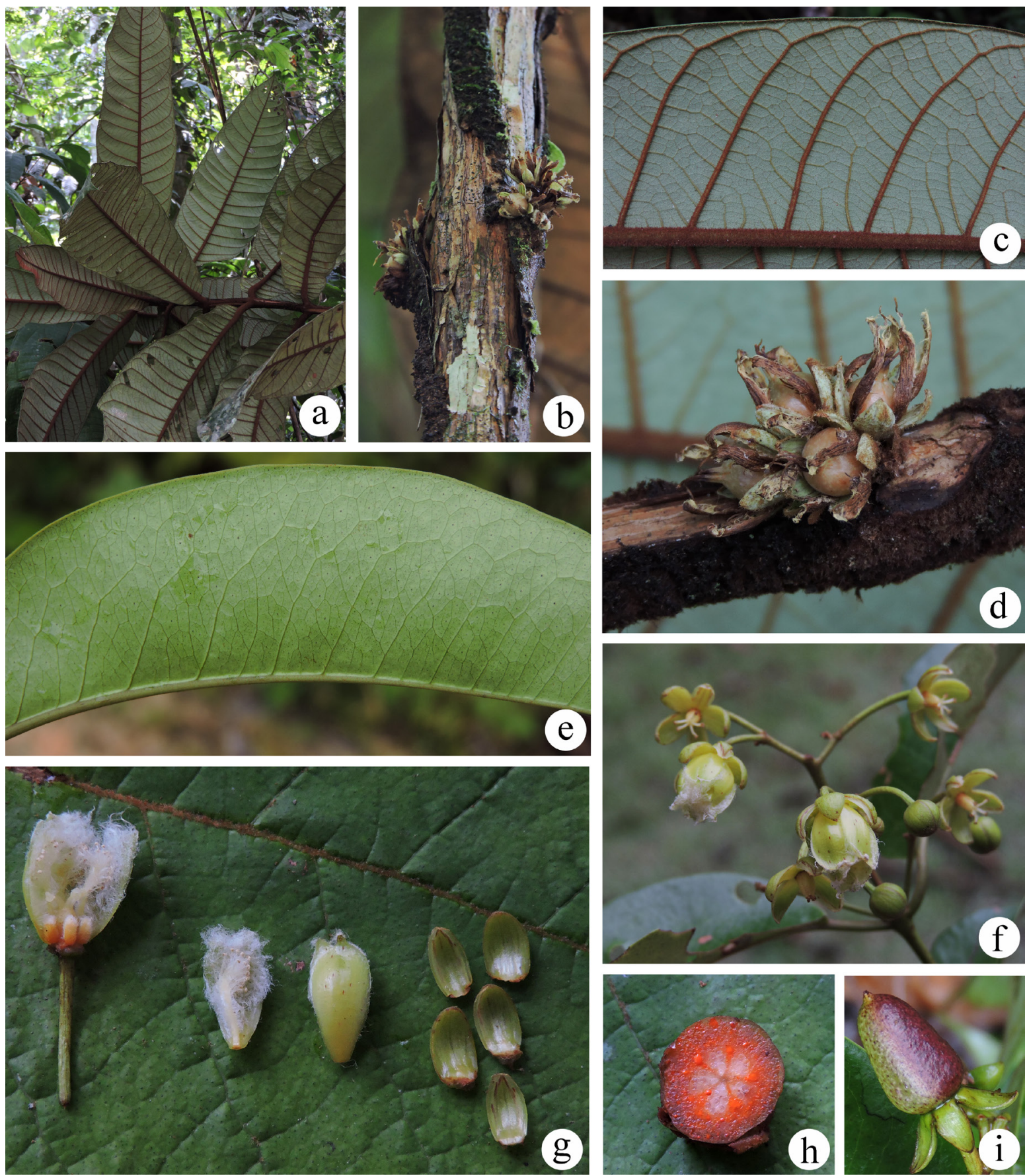

Figura 2. a-d. Erythroxylum passerinum Mart. a. Estípula, vista abaxial. b. Folha, vista adaxial. c. Flor com corola removida. d. Drupa em secção transversal. e-h. Erythroxylum rimosum O.E.Schulz. e. Hábito. f. Estípula, vista abaxial. g. Folha, vista adaxial. h. Botão floral. i-1. Erythroxylum simonis Plowman. i. Estípula, vista abaxial. j. Folha, vista adaxial. k. Flor com corola removida. 1. Drupa em secção transversal. Ilustração elaborada por Felipe Martins.

Figure 2. a-d. Erythroxylum passerinum Mart. a. Stipule, ventral view. b. Leaf blade, dorsal view. c. Flower, corolla removed. d. Drupe in cross-section. e-h. Erythroxylum rimosum O.E.Schulz. e. Habit. f. Stipule, ventral view. g. Leaf blade, dorsal view. h. Flower bud. i-1. Erythroxylum simonis Plowman. i. Stipule, ventral view. j. Leaf blade, dorsal view. k. Flower, corolla removed. 1. Drupe in cross-section. Drawing by Felipe Martins. 
Cabeça de Boi, 11-II-2010 (fl.), M.R. Barbosa 3302 (JPB); ibidem, Mata Cabeça de Boi, nas proximidades da trilha principal, 6 $6^{\circ} 42^{\prime} 19^{\prime} \mathrm{S}, 35^{\circ} 10^{\prime} 36^{\prime} \mathrm{W}, 103 \mathrm{~m}$, 28-II-2013 (fl.), J.L. Costa-Lima et al. 890 (JPB, UFP, UFRN). Mun. Rio Tinto, SEMA III, Mata do Maracujá, 31-I-1989 (fl.), L.P. Félix s.n. (JPB 8017); ibidem, 18-V-1989 (fr.), C.A.B. de Miranda s.n. (JPB 22026); ibidem, 23-V-1990 (fl.), L.P. Félix 3031 (JPB); ibidem, 17-I-2009 (fl.), M.E.M. Fortunato 11 (JPB); ibidem, 17-I-2009 (fl., fr.), M.E.M. Fortunato 12 (JPB); ibidem, 17-I-2009 (fl.), M.E.M. Fortunato 17 (JPB); ibidem, 18-III-2009 (fr.), M.E.M. Fortunato 22 (JPB); ibidem, 3-II-2010 (fl.), M.E.M. Fortunato 40 (JPB); ibidem, 3-III-2010 (fl., fr.), M.E.M. Fortunato 40 (JPB); ibidem, 23-III-2010 (fr.), P.C. Gadelha Neto et al. 2765 (JPB, RB).

É reconhecida pelas folhas cartáceas, em geral com lâminas largamente elípticas, opacas e pelas drupas com pirênio sulcado longitudinalmente em secção transversal.

Ocorre nos Estados do Rio Grande do Norte ao Rio de Janeiro vegetando em áreas de restinga, tabuleiros litorâneos e manguezais (Loiola \& CostaLima 2015). Na REBIO Guaribas é encontrada em todas as três áreas: SEMA 1, 2 e 3, especialmente na orla das matas e na transição mata-tabuleiro. A espécie é encontrada em estádio fértil na maior parte do ano.

\section{Erythroxylum rimosum O.E.Schulz in Engl.,} Pflanzenr. 4 (134): 53. 1907.

Figura 2 e-h

Subarbustos, 0,4-1,5 m alt.; ramos com lenticelas ausentes a raras, acinzentados. Estípulas 1-2,4 mm compr., coléteres inconspícuos. Pecíolo 1,2-2,4 mm compr.; folhas com lâmina 3-7 × 1,2-4 cm, oblongas a obovadas, coriáceas, opacas, base aguda, margem finamente revoluta, ápice obtuso. Fascículos com 1-5 flores por nó; pedicelo 4,5-7,8 mm compr.; cálice com prefloração contorcida, lobos 1,4-2,2 × 1,2-1,6 mm, oblongos, ápice apiculado; pétalas 3,4-4,5 mm compr.; tubo estaminal 1,2-1,5 mm compr.; filetes 1,2-2,2 mm compr.; estiletes 2-2,5 mm compr., livres; ovário 1,5-2 $\times 1,2-1,6 \mathrm{~mm}$, ovoide. Drupas com pirênio 10-13 × 4,2-5,5 mm, ovoide, cilíndrico em secção transversal.

Material examinado: BRASIL. Paraíba: Mun. Mamanguape, Reserva Biológica Guaribas, Sema I, 5-IV-1989 (fl.), L.P. Félix 3554 (JPB); ibidem, Capim Azul, 7-III-1990 (fl.), L.P. Félix 2792 (JPB); ibidem, Área II, 9-III-2002 (fr.), M.R. Barbosa 2302 (JPB); ibidem, Área I, Capim Azul, 06 41 '54"'S, 3507'20”'W, 19-VIII-2002 (st.), A.C. Sevilha \& G. Pereira-Silva 2236 (CEN); ibidem, 06 41'51'"S, 3507'22”'W, 25-XI-2002 (fr.), A.C. Sevilha \& G. Pereira-Silva 2524 (CEN); ibidem, 25-XI-2002 (fr.), A.C. Sevilha \& G. Pereira-Silva 2526 (CEN); ibidem, Área I, Caiana, 2-II-2003 (fl.), M.R. Barbosa 2647 (JPB); ibidem, próximo a Trilha Caiana, 14-VIII-2008 (fl., fr.), F.O. Silva 3 (JPB); ibidem, 17-XII-2009 (fl.), F.O. Silva 51 (JPB); ibidem, 12-I-20010 (fl., fr.), F.O. Silva 84 (JPB); ibidem, 06 42 '02'S, 3507'23'”W, 28-II-2013 (fr.), J.L. Costa-Lima et al. 888 (HUEFS, JPB, UFP, UFRN); ibidem, 28-II-2013 (fr.), J.L. Costa-Lima et al. 889 (JPB, UFP, UFRN).

Apresenta como caracteres diagnósticos as folhas com lâminas oblongas a obovadas, de textura coriácea e com margem finamente revoluta, além do cálice com prefloração contorcida e lobos oblongos com ápice apiculado.

Com registros nos Estados do Piauí, Ceará, Rio Grande do Norte, Paraíba, Pernambuco e Sergipe (Loiola et al. 2007, Costa-Lima et al. 2014, CostaLima \& Alves 2015b), Erythroxylum rimosum habita formações savânicas no Nordeste do Brasil, em áreas de carrasco e nos tabuleiros litorâneos. $\mathrm{Na}$ REBIO Guaribas foi encontrada na SEMA 1, onde predomina a vegetação de tabuleiro litorâneo. Baseado nos materiais analisados, apresenta flores e frutos de novembro a abril.

Destaca-se que o nome Erythroxylum suberosum var. denudatum O.E. Schulz, amplamente aplicado as amostras desta espécie, inclusive à maioria dos materiais provenientes da REBIO Guaribas, foi recentemente sinonimizado sob E. rimosum por CostaLima et al. (2015).

3. Erythroxylum simonis Plowman, Brittonia 38(3): 189. 1986.

Figura 2 i-1

Arbustos, 1,5-4 m alt.; ramos lenticelados, castanho-acinzentados. Estípulas 1,5-2,2 mm compr., coléteres conspícuos e levemente vilosos. Pecíolo 1-2,4 mm compr.; folhas com lâmina 3,5-5,2 × 1,5-2,6 cm, elípticas a estreitamente elípticas, membranáceas, lúcidas na face adaxial, base aguda, margem plana, ápice acuminado. Fascículos com 1-4 flores por nó; pedicelo 2-3 mm compr.; cálice com prefloração valvar, lobos 0,8-1 × 0,5-0,8 mm, triangulares, ápice agudo; pétalas 2-2,5 mm compr.; tubo estaminal 1-1,5 mm compr.; filetes 1-1,6 mm 
compr.; estiletes 0,8-2,5 mm compr., livres; ovário 1-1,2 $\times 0,6-0,8 \mathrm{~mm}$, obovoide. Drupas com pirênio $7-8,5 \times 5,5-6,5 \mathrm{~mm}$, ovoide, cilíndrico em secção transversal.

Material examinado: BRASIL. PAraíba: Mun. Mamanguape, Mata Cabeça de Boi, nas proximidades da trilha principal, 6042'19' $\mathrm{S}, 35^{\circ} 10^{\prime} 36^{\prime \prime} \mathrm{W}, 103 \mathrm{~m}$, 28-II-2013 (fl.), J.L. Costa-Lima et al. 891 (RB, UFP, UFRN).

Material adicional examinado: BRASIL. PARAÍBA: Mun. João Pessoa, Costa do Sol, 4-V-1991 (fr.), M.R. Barbosa 1218 (JPB).

Pode ser reconhecida, principalmente, por ser um arbusto delicado no interior das matas, pelas suas folhas com lâminas lúcidas na face adaxial, flores com tubo estaminal maior que os lobos do cálice e frutos com pirênio cilíndrico em secção transversal.

Erythroxylum simonis foi registrada em áreas de Mata Atlântica nos Estados do Ceará, Rio Grande do Norte, Paraíba, Pernambuco e Sergipe (Loiola $\&$ Costa-Lima 2015), onde ocorre no sub-bosque das matas de restinga, florestas estacionais de terras baixas e nos brejos de altitude. Na REBIO Guaribas foi observada como elemento frequente no interior das matas da SEMA 2. Coletada com flores no fim de fevereiro, com floração observada até março.

\section{Conclusão}

Foram reconhecidas três espécies de Erythroxylum na REBIO Guaribas, ressaltando, assim, a importância do estudo mais acurado dos acervos e de coletas adicionais para confirmar a real riqueza taxonômica da família na área estudada, esta, até então, superestimada. Neste estudo, além de uma melhor resolução taxonômica das amostras e com informações sobre sinonímia, os acervos analisados se encontram com nomes devidamente atualizados. As espécies foram diferenciadas, principalmente, pela forma e textura das lâminas foliares, presença de coléteres nas estípulas, prefloração do cálice e forma dos lobos e morfologia do pirênio, tornando-se possíveis identificações futuras com base em amostras em estádio vegetativo ou em diferentes estádios reprodutivos. Embora nenhuma das três espécies aqui tratadas se encontre sob risco de ameaça em escala global, todas ocorrem em ambientes fortemente ameaçados e alterados, como as restingas e os tabuleiros litorâneos. Desse modo, evidenciamos a importância local de uma área protegida como a REBIO Guaribas para a manutenção e salvaguarda de espécies e dos ecossistemas nessa porção extremamente fragmentada da Mata Atlântica.

\section{Agradecimentos}

Os autores agradecem ao ICMBio e à equipe da Reserva Biológica Guaribas pela autorização de coleta e apoio logístico durante as atividades de campo; à Dra. Maria Regina de Vasconcellos Barbosa e ao Dr. William Wayt Thomas pelo auxílio durante a preparação do manuscrito; e ao Dr. Earl Chagas pela leitura da versão inicial do manuscrito. O primeiro autor agradece também a Coordenação de Aperfeiçoamento de Pessoal de Nível Superior (CAPES) pela bolsa concedida no período 2012-2014 (PROTAX - Edital MCT/CNPq/ MEC/CAPES $n^{\circ}$ 52/2010), junto ao Programa de Pós-Graduação em Biologia Vegetal da Universidade Federal de Pernambuco, e a U.S. National Science Foundation, Velux Stiftung, Beneficia Foundation e Conselho Nacional de Desenvolvimento Científico e Tecnológico (CNPq) pelo financiamento das atividades de campo.

\section{Literatura citada}

Bachman, S., Moat, J., Hill, A.W., de la Torre, J. \& Scott, B. 2011. Supporting Red List threat assessments with GeoCAT: geospatial conservation assessment tool (Version BETA). In: V. Smith \& L. Penev (eds.). E-Infrastructures for Data Publishing in Biodiversity Science. ZooKeys 150: 117-126.

Barbosa, M.R.V., Thomas, W.W., Zárate, E.L.P., Lima, R.B., Agra, M.F., Lima, I.B., Pessoa, M.C.R., Lourenco, A.R.L., Delgado Junior, G.C., Pontes, R.A.S., Chagas, E.C.O., Viana, J.L., Gadelha Neto, P.C., Araújo, C.M.L.R., Araújo, A.A.M., Freitas, G.B., Lima, J.R., Silva, F.O., Vieira, L.A.F., Pereira, L.A., Costa, R.M.T., Duré, R.C. \& Sá, M.G.V. 2011. Checklist of the Vascular Plants of the Guaribas Biological Reserve, Paraíba, Brazil. Revista Nordestina de Biologia 20: 79-106.

Costa-Lima, J.L., Loiola, M.I.B. \& Jardim, J.G. 2014. Erythroxylaceae no Rio Grande do Norte, Brasil. Rodriguésia 65: 659-671.

Costa-Lima, J.L. \& Alves, M. 2015a. Three new species in Brazilian Erythroxylum (Erythroxylaceae). Phytotaxa 192: 97-104.

Costa-Lima, J.L. \& Alves, M. 2015b. Flora da Usina São José, Igarassu, Pernambuco: Erythroxylaceae. Rodriguésia 66: 285-295.

Costa-Lima, J.L., Loiola, M.I.B. \& Alves, M. 2015. News synonyms and lectotypifications in Brazilian Erythroxylum (Erythroxylaceae). Phytotaxa 201: 100-102. 
Daly, D. 2004. Erythroxylaceae. In: N. Smith, S.A. Mori, A. Henderson, D.W. Stevenson \& S. Heald (eds.). Flowering Plants of Neotropics. The New York Botanical Garden, Princeton University Press, New York, pp. 143-145.

Harris, J.G. \& Harris, M.W. 2001. Plant Identification Terminology: an illustrated glossary. 2. ed. Spring Lake Publishing, Spring Lake, Utah.

IBAMA (Instituto Brasileiro do Meio Ambiente e dos Recursos Naturais Renováveis). 2003. Plano de Manejo, Fase 2, da Reserva Biológica de Guaribas. Ministério do Meio Ambiente/IBAMA, Brasília. Disponível em http://www.icmbio.gov.br/portal/images/ stories/imgs-unidades-coservacao/rebio_guaribas.pdf (acesso em 23-I-2018).

IUCN (International Union for the Conservation of Nature). 2012. IUCN Red List Categories and Criteria: version 3.1. Second edition. Gland, Switzerland and Cambridge: IUCN. Disponível em http://cmsdocs. s3.amazonaws.com/keydocuments/Categories_and Criteria_en_359 web\%2Bcover\%2Bbckcover.pdf (acesso em 23-I-2018).

Loiola, M.I.B., Agra, M.F., Baracho, G.S. \& Queiroz, R.T. 2007. Flora da Paraíba, Brasil: Erythroxylaceae Kunth. Acta Botanica Brasilica 21: 473-487.

Loiola, M.I.B. \& Costa-Lima, J.L. 2015. Erythroxylaceae. In: Lista de Espécies da Flora do Brasil. Jardim Botânico do Rio de Janeiro. Disponível em http://floradobrasil. jbrj.gov.br/jabot/floradobrasil/FB111 (acesso em 23-I-2018).

Martius, C.F.P. von. 1840. Beiträge zur kenntnis der gattung Erythroxylon. Abhandlungen der MathematischPhysikalischen Classe der Königlich Bayerischen Akademie der Wissenschaften 3: 281-412.

Melo, J.I.M. \& Vieira, D.D. 2017. Flora da Reserva Biológica Guaribas, PB, Brasil: Boraginaceae. Hoehnea 44: 407-414.

Mori, S.A., Silva, L.A.M., Lisboa, G. \& Corandin, L. 1989. Manual de manejo do herbário fanerogâmico. 2 ed. Centro de Pesquisas do Cacau, Ilhéus.
Pereira, M.S. \& Barbosa, M.R.V. 2004. A família Rubiaceae na Reserva Biológica Guaribas, Paraíba, Brasil. Subfamílias Antirheoideae, Cinchonoideae e Ixoroideae. Acta Botanica Brasilica 18: 305-318.

Pereira, M.S. \& Barbosa, M.R.V. 2006. A família Rubiaceae na Reserva Biológica Guaribas, Paraíba, Brasil. Subfamília Rubioideae. Acta Botanica Brasilica 20: 455-470.

Plowman, T.C. 1986. Four new species of Erythroxylum (Erythroxylaceae) from northeastern Brazil. Brittonia 38: $189-200$.

Plowman, T.C. 1987. Ten new species of Erythroxylum (Erythroxylaceae) from Bahia, Brazil. Fieldiana, Botany 19: 1-41.

Plowman, T.C. \& Berry, P.E. 1999. Erythroxylaceae. In: J.A. Steyermark, P.E. Berry, K. Yatskievych \& B. Holst (eds.). Flora of Venezuelana Guayana. v. 5. Missouri Botanical Garden Press, St. Louis, pp. 59-71.

Plowman, T.C. \& Hensold, N. 2004. Names, types, and distribution of neotropical species of Erythroxylum (Erythroxylaceae). Brittonia 56: 1-53.

Ranta, P., Bolm, T., Niemela, J., Joensuu, E. \& Siitonen, M. 1998. The fragmented Atlantic rain forest of Brazil: size, shape and distribution of forest fragments. Biodiversity and Conservation 7: 385-403.

Schulz, O.E. 1907. Erythroxylaceae. In: A. Engler (ed.). Das Pflanzenreich: regni vegetabilis conspectus. Verlag von Wilhelm Engelmann, Leipzig, IV, 134 (Heft 39), pp. 1-176.

Stearn, W.T. 1992. Botanical Latin. 4. ed. David \& Charles Book, Redwood Press, England Ltd., London.

Thiers, B. 2018 (continuamente atualizado). Index Herbariorum: a global directory of public herbaria and associated staff. New York Botanical Gardens Virtual Herbarium. Disponível em http://sweetgum.nybg.org/ ih (acesso em 23-I-2018).

Thomas, W.W. \& Barbosa, M.R.V. 2008. Natural vegetation types in the Atlantic coastal of Northeastern Brazil. In: W.W. Thomas (ed.). The Atlantic Coastal Forest of Northeastern Brazil. New York Botanical Garden Press, New York, v. 100, pp. 6-20. 


\section{ERRATA}

No artigo "Flora da Reserva Biológica Guaribas, Estado da Paraíba, Brasil: Erythroxylaceae", com número de DOI: http://dx.doi.org/10.1590/2236-8906-84/2017, publicado no periódico Hoehnea, 45(3): 372-378. na página 373:

Onde se lia:

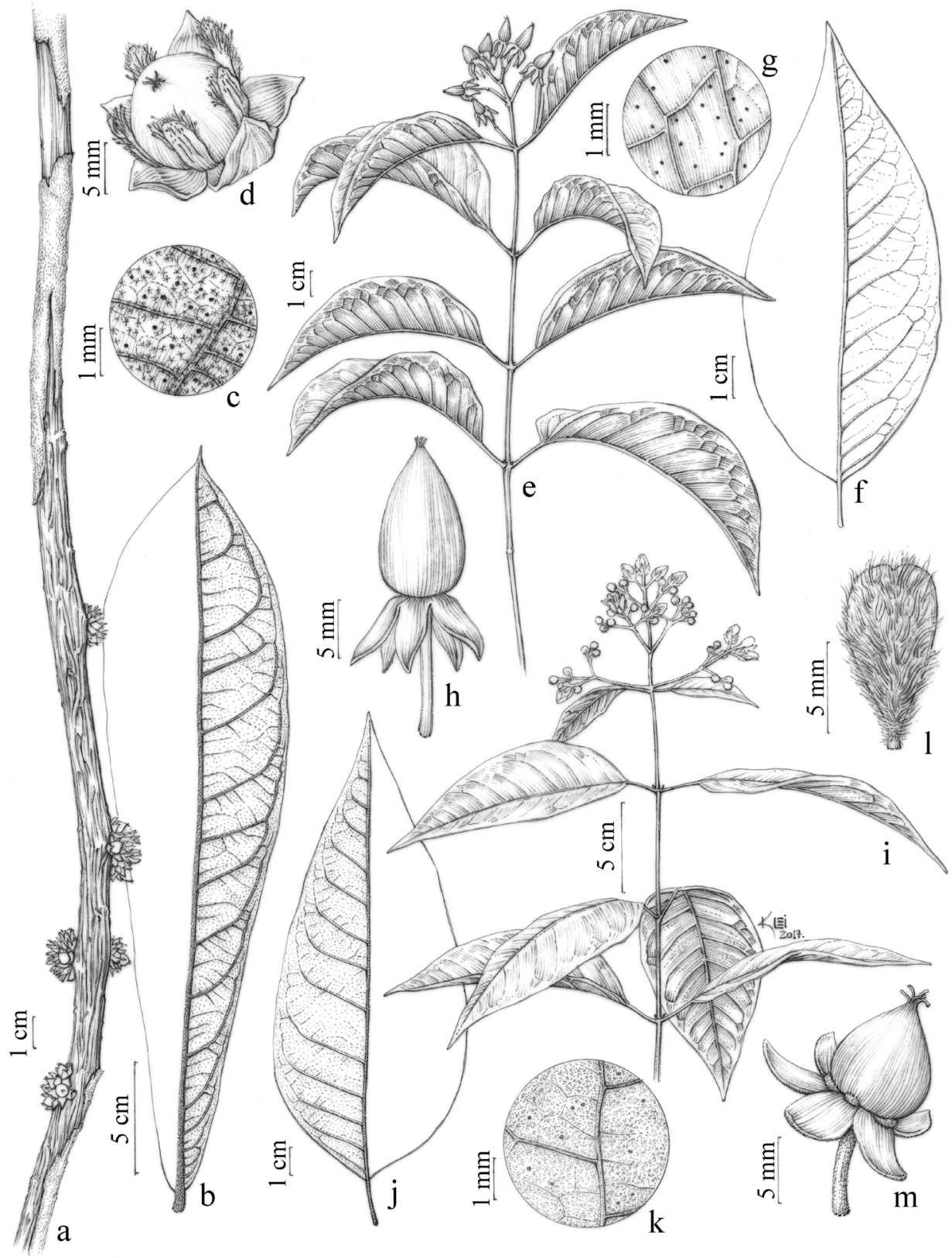

Figura 1. Mapa de localização da Reserva Biológica (REBIO) Guaribas, Estado da Paraíba, Nordeste do Brasil, e suas áreas. Mapa elaborado por Rafael Barbosa da Silva.

Figure 1. Location map of the Guaribas Biological Reserve (REBIO), State of Paraíba, northeastern Brazil, and its areas. Map by Rafael Barbosa da Silva. 


\section{Leia-se:}

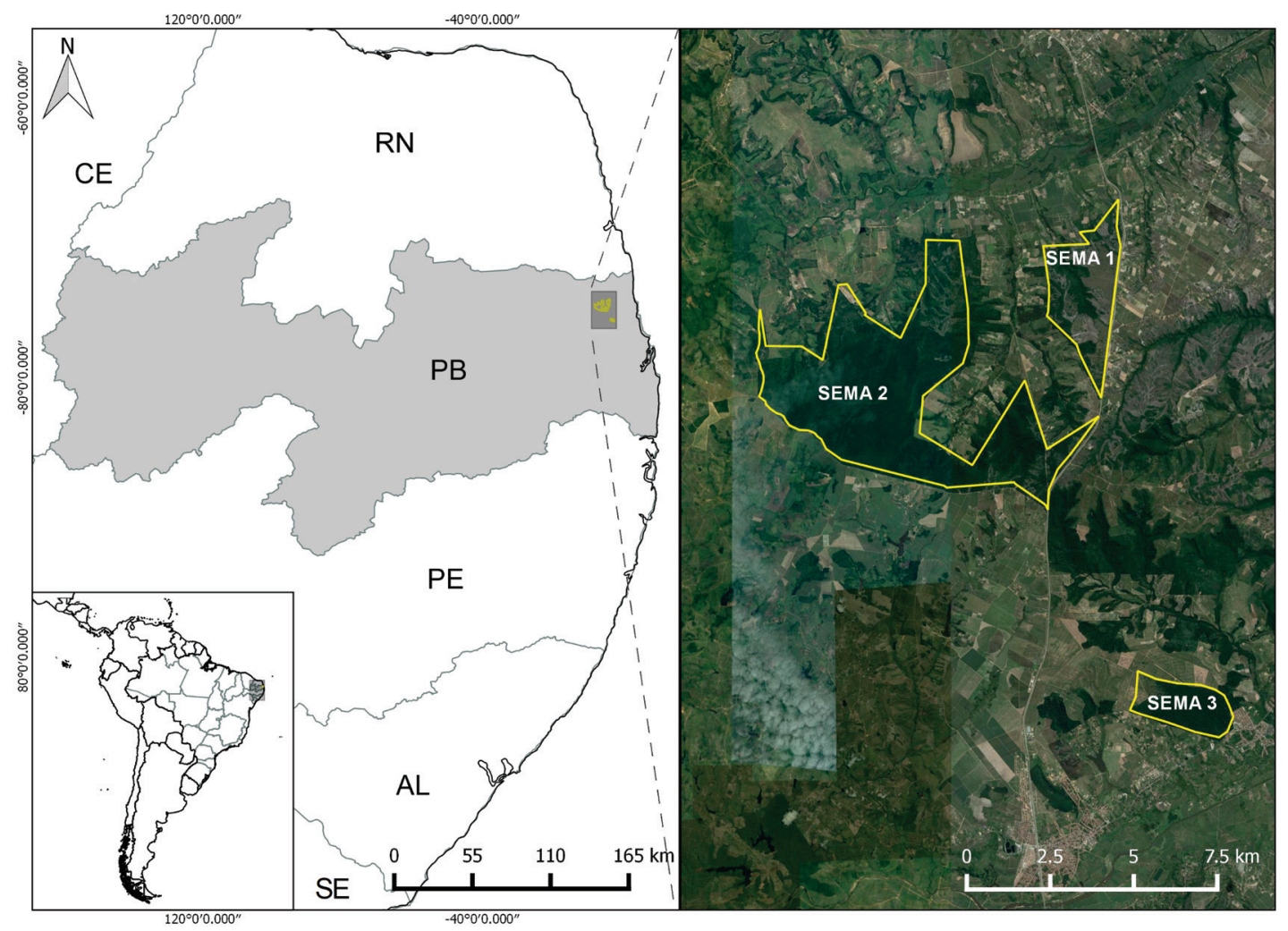

Figura 1. Mapa de localização da Reserva Biológica (REBIO) Guaribas, Estado da Paraíba, Nordeste do Brasil, e suas áreas. Mapa elaborado por Rafael Barbosa da Silva.

Figure 1. Location map of the Guaribas Biological Reserve (REBIO), State of Paraíba, northeastern Brazil, and its areas. Map by Rafael Barbosa da Silva. 
na página 375:

Onde se lia:
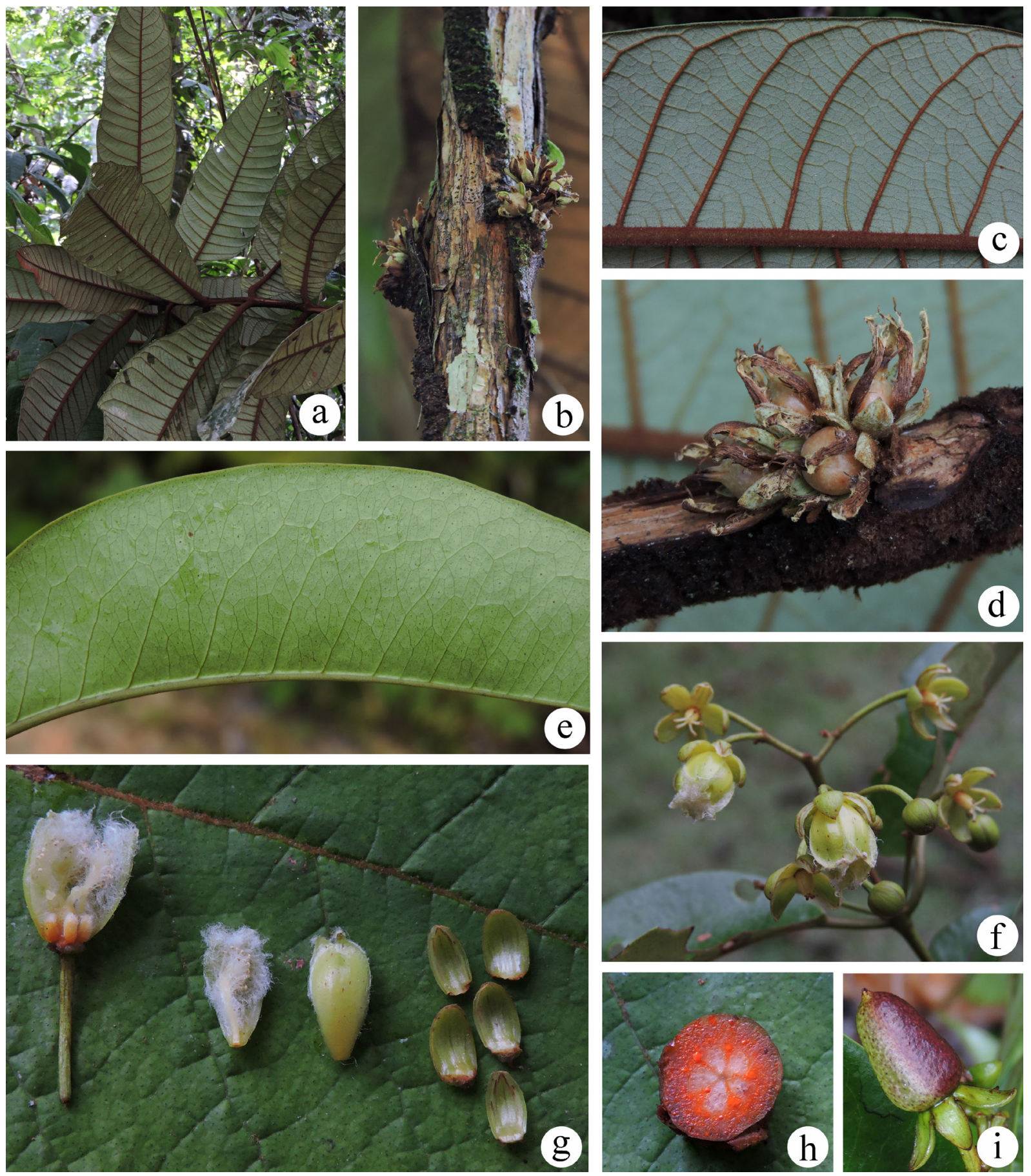

Figura 2. a-d. Erythroxylum passerinum Mart. a. Estípula, vista abaxial. b. Folha, vista adaxial. c. Flor com corola removida. d. Drupa em secção transversal. e-h. Erythroxylum rimosum O.E.Schulz. e. Hábito. f. Estípula, vista abaxial. g. Folha, vista adaxial. h. Botão floral. i-1. Erythroxylum simonis Plowman. i. Estípula, vista abaxial. j. Folha, vista adaxial. k. Flor com corola removida. 1. Drupa em secção transversal. Ilustração elaborada por Felipe Martins.

Figure 2. a-d. Erythroxylum passerinum Mart. a. Stipule, ventral view. b. Leaf blade, dorsal view. c. Flower, corolla removed. d. Drupe in cross-section. e-h. Erythroxylum rimosum O.E.Schulz. e. Habit. f. Stipule, ventral view. g. Leaf blade, dorsal view. h. Flower bud. i-1. Erythroxylum simonis Plowman. i. Stipule, ventral view. j. Leaf blade, dorsal view. k. Flower, corolla removed. 1. Drupe in cross-section. Drawing by Felipe Martins. 


\section{Leia-se:}
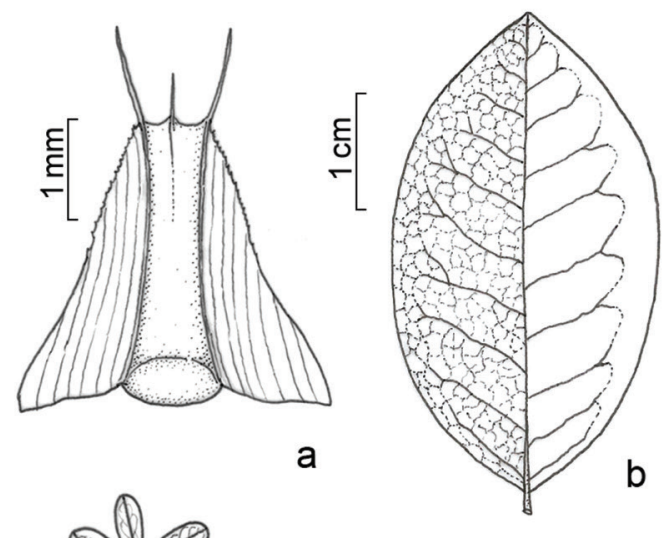
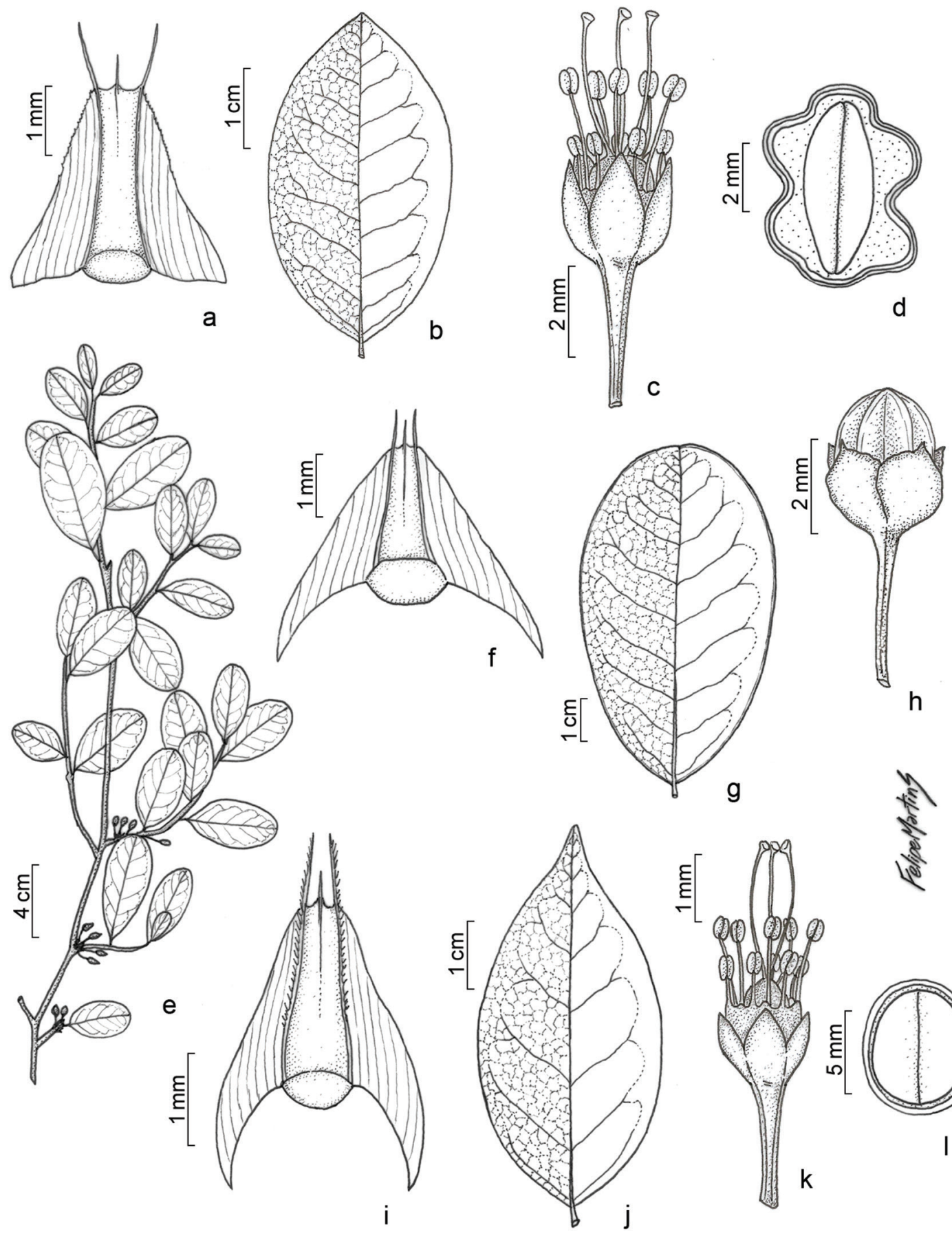

h
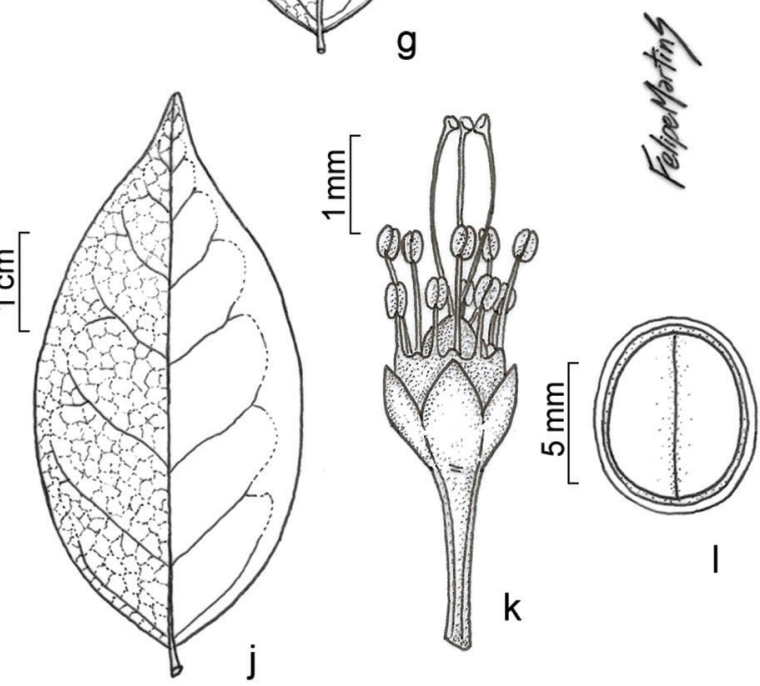

k

Figura 2. a-d. Erythroxylum passerinum Mart. a. Estípula, vista abaxial. b. Folha, vista adaxial. c. Flor com corola removida. d. Drupa em secção transversal. e-h. Erythroxylum rimosum O.E.Schulz. e. Hábito. f. Estípula, vista abaxial. g. Folha, vista adaxial. h. Botão floral. i-1. Erythroxylum simonis Plowman. i. Estípula, vista abaxial. j. Folha, vista adaxial. k. Flor com corola removida. 1. Drupa em secção transversal. Ilustração elaborada por Felipe Martins.

Figure 2. a-d. Erythroxylum passerinum Mart. a. Stipule, ventral view. b. Leaf blade, dorsal view. c. Flower, corolla removed. d. Drupe in cross-section. e-h. Erythroxylum rimosum O.E.Schulz. e. Habit. f. Stipule, ventral view. g. Leaf blade, dorsal view. h. Flower bud. i-l. Erythroxylum simonis Plowman. i. Stipule, ventral view. j. Leaf blade, dorsal view. k. Flower, corolla removed. 1. Drupe in cross-section. Drawing by Felipe Martins. 